\title{
Review
}

\section{The role of BDNF in depression on the basis of its location in the neural circuitry}

\author{
Hui YU, Zhe-yu CHEN*
}

Department of Neurobiology, Shandong Provincial Key Laboratory of Mental Disorders, School of Medicine, Shandong University, Jinan 250012, China

\begin{abstract}
Depression is one of the most prevalent and life-threatening forms of mental illnesses and the neural circuitry underlying depression remains incompletely understood. Most attention in the field has focused on hippocampal and frontal cortical regions for their roles in depression and antidepressant action. While these regions no doubt play important roles in the mental illness, there is compelling evidence that other brain regions are also involved. Brain-derived neurotrophic factor (BDNF) is broadly expressed in the developing and adult mammalian brain and has been implicated in development, neural regeneration, synaptic transmission, synaptic plasticity and neurogenesis. Recently BDNF has been shown to play an important role in the pathophysiology of depression, however there are controversial reports about the effects of BDNF on depression. Here, we present an overview of the current knowledge concerning BDNF actions and associated intracellular signaling in hippocampus, prefrontal cortex, nucleus accumbens (NAc) and amygdala as their relation to depression.
\end{abstract}

Keywords: BDNF; depression; antidepressant; neural circuitry

Acta Pharmacologica Sinica (2011) 32: 3-11; doi: 10.1038/aps.2010.184; published online 6 Dec 2010

\section{Introduction}

Depression is a clinically and biologically heterogeneous disease, with $10 \%-30 \%$ of women and $7 \%-15 \%$ of men likely to suffer from depression in their life-time ${ }^{[1]}$. The symptoms of depression are the loss of interest or pleasure in virtually all activities (anhedonia) and a long-lasting depressed mood, feelings of guilt, anxiety, and recurrent thoughts of death and suicide $^{[2]}$. The genetic contribution to the manifestation of depression has been estimated as $40 \%-50 \%{ }^{[3]}$. However, combinations of multiple genetic factors may be involved in the development of depression, because a defect in a single gene usually fails to induce the expression of multifaceted symptoms of depression ${ }^{[4]}$. Also, various non-genetic factors such as stress, affective trauma, viral infection, and neurodevelopmental abnormalities increase the complexity of the pathogenesis of the disease. Thus, extensive studies have led to a variety of hypotheses for the molecular mechanism of depression, but a definite pathogenic mechanism has yet to be defined. The objective of this review is to investigate the contribution of brain-derived neurotrophic factor (BDNF) and its intracellular signaling in different brain regions to depression and antide-

\footnotetext{
* To whom correspondence should be addressed. E-mail zheyuchen@sdu.edu.cn

Received 2010-07-05 Accepted 2010-09-24
}

pressant treatments.

\section{Neurobiology of depression}

As a basic understanding of depression the monoamine hypothesis was formulated in the mid 1960s based on the antidepressant efficacy of the monoamine reuptake inhibitors, monoamine oxidase inhibitors and the depressogenic effects of reserpine as a monoamine depleter ${ }^{[5]}$. This hypothesis suggests a deficiency or imbalances in the monoamine neurotransmitters, such as serotonin (5-HT), dopamine and norepinephrine (NE), as the cause of depression. Among therapeutic agents, antidepressants including tricyclics, monoamine oxidase inhibitors and selective serotonin reuptake inhibitors (SSRIs) exert their therapeutic action through their ability to increase the synaptic content of monoamine neurotransmitters $^{[6]}$. However, antidepressants exert their therapeutic action only after chronic treatment, indicating that enhanced 5-HT or NE neurotransmission per se is not responsible for the clinical actions of these drugs. Second, antidepressants are effective in less than $50 \%$ of patients ${ }^{[2]}$, which suggests that additional biological substrates could provide potential therapeutic targets.

Based on clinical and animal studies it has been suggested that depression are associated with neuronal atrophy and neuronal cell loss, especially in the hippocampus and cerebral cortex $^{[7]}$. Therefore the neurotrophic factors are recognized as an 
important new lead in the quest for a deeper understanding of depression and the mechanisms of antidepressants effect ${ }^{[8]}$. The neurotrophic hypothesis of depression states that a deficiency in neurotrophic support may contribute to hippocampal pathology during the development of depression, and the reversal of this deficiency by antidepressant treatments may contribute to the resolution of depressive symptoms. Of various neurotrophins, most studies have focused on BDNF, one of the most prevalent neurotrophic factors in adult brain.

\section{BDNF and depression}

Studies in humans have shown decreased plasma levels of BDNF in bipolar disorder, manic and depressed patients ${ }^{[9,10]}$. Many preclinical and clinical studies provide direct evidence suggesting that modulation in expression of BDNF could be involved in behavioral phenomenon associated with depression. Recently, a variant in the human BDNF gene, which leads to a valine to methionine change in the pro-domain of the BDNF protein (position 66), was found to decrease activity-dependent secretion of $\mathrm{BDNF}^{[11,12]}$ and be associated with increased susceptibility to neuropsychiatric disorders including depression, anxiety-related dysfunction and bipolar disorder ${ }^{[13-17]}$. People with the Met allele have been found to have a relatively small hippocampus and poor hippocampusdependent memory function ${ }^{[18]}$. In our previous work, we have generated a BDNF knock-in mouse containing the BDNF Val66Met polymorphism, which provided a valuable tool to assess the in vivo consequence of BDNF ${ }^{\text {Met }}$ polymorphism ${ }^{[19]}$. We found that $\mathrm{BDNF}^{\mathrm{Met} / \mathrm{Met}}$ mice showed increased anxietyrelated behaviors, which could not be reversed by fluoxetine treatment ${ }^{[19]}$. Furthermore, we found BDNF ${ }^{\text {Met/Met }}$ mice had decreased ventromedial prefrontal cortex volume and displayed aversive memory extinction deficit ${ }^{[20]}$. These studies suggest that the BDNF Val66Met polymorphism might be at risk to develop smaller prefrontal cortex and hippocampus and thus lead to susceptibility to mood disorders, which strengthens the hypothesis that BDNF plays an important role in depression.

The role of BDNF in depression has also been supported by the hypothesis that BDNF mediates the action of antidepressant. The most widely accepted hypothesis assuming that antidepressants restore the compromised neurotransmission in mainly noradrenergic and serotoninergic system has dominated our thinking of antidepressant action. Indeed, speaking of depression, a key question has always been why therapeutic responses with antidepressants could only be achieved after at least 2-3 weeks of treatment, whereas antidepressants alter synaptic monoamine levels within hours. Since the monoaminergic hypothesis cannot fully explain this delay of antidepressant effect, it has been suggested that this delay is required in order to produce neuroadaptive mechanisms that may enhance neuronal plasticity and resilience ${ }^{[21,22]}$. Along this line of reasoning, several studies have shown that BDNF may mediate the therapeutic action of antidepressants ${ }^{[23-25]}$. There is a plethora of evidence documenting that antidepressant treatments, including SSRIs and electroconvulsive shock
(ECS) increase the expression of BDNF and tropomyosin receptor kinase B (TrkB) in the hippocampus in animal models. These effects are dependent on chronic administration of antidepressant therapy, consistent with the time course of antidepressant treatments ${ }^{[26,27]}$. This suggests that the regulations of BDNF and TrkB are involved in the pathology and development of depression and antidepressant treatments.

However, although BDNF exerts antidepressant-like effects in hippocampus, its actions might be different or even opposite, in different brain regions. The best example is the ventral tegmental area-nucleus accumbens (NAc) dopaminergic reward circuit, in which chronic stress increases BDNF expression $^{[28]}$. Local BDNF infusion into NAc exerts a prodepressionlike effect in the forced swim test, and blockade of BDNF function in NAc exerts an antidepressant-like effect ${ }^{[28]}$. Thus the differential role of BDNF in depression might be attributed to its location in the depression neural circuits. In the following parts of the review, we would like to summarize the local contribution of BDNF/TrkB signaling to depression on the basis of its location in the neural circuitry.

\section{Neural circuitry of depression}

Disturbances in the detection of, response to, and interpretation of emotion are common in depression. The affective fronto-limbic circuitry including the prefrontal cortices, the cingulate cortex, several limbic structures including the hippocampus, amygdala, lower brainstem structures and the basal ganglia are highly involved in mediating these stages of emotion processing, and evidence indicates that these regions show structural and functional alterations in depression ${ }^{[29]}$.

A greater understanding of the neural circuitry underlying normal mood and abnormalities in mood has been identified as one of the critical needs in the field of depression research. The hippocampus and frontal cortex are implicated in learning and memory, attention and impulse control, which suggests they may mediate cognitive aspects of depression, such as memory impairments and feelings of hopelessness, guilt, doom, and suicidality ${ }^{[2,30]}$. The striatum (particularly the NAc) and amygdala, and related brain areas, are important in emotional memory, and could as a result mediate the anhedonia, anxiety, and reduced motivation that predominated in many patients ${ }^{[31]}$. Hypothalamic abnormalities likely contribute to altered appetite and autonomic symptoms. Thalamic and brainstem dysregulation contribute to altered sleep and arousal states ${ }^{[32]}$. Of course, these various brain areas cannot be thought of as distinct, they operate in a series of highly interacting parallel circuits, which perhaps begins to formulate a neural circuitry involved in depression.

Brain imaging studies of depressed patients indicate a significant reduction in hippocampal volume compared with healthy subjects ${ }^{[33,34]}$. Magnetic resonance imaging (MRI) studies consistently show that the ventral prefrontal cortex is reduced in size in adult patients with major depressive disorder (MDD) compared with healthy controls. Postmortem data support this finding, and suggest that fewer glia cells may contribute to the overall reduced size of the region ${ }^{[35]}$. To the 
striatum, MRI and postmortem studies showed volumetric reductions in the caudate and the ventral region of the striatum in individuals with depression ${ }^{[36]}$, but other studies have found no differences ${ }^{[37]}$. In the amygdale, findings are not entirely consistent across studies. It has been reported that amygdala volumes were increased in patients during first episode with MDD, but patients with recurring MDD had smaller amygdala ${ }^{[38]}$. Other study reported that adult patients with MDD have increased amygdala volume than healthy comparison subjects ${ }^{[39]}$. Thus, inconsistencies in the data may relate to stage of the disorder. In summary, the anatomical data suggest that adult MDD is associated with reduced hippocampus, ventral prefrontal cortex volume and altered amygdala volume in comparison with healthy controls.

\section{BDNF signaling in hippocampus involved in depression}

The hippocampus, a key structure for the encoding of emotionally relevant data into memory, interacts with the amygdala to provide input regarding the context in which stimuli occur ${ }^{[40]}$. In addition, the hippocampus plays an inhibitory role in the regulation of the amygdala and hypothalamic- pituitaryadrenal (HPA) axis activity, which is the major mediator of systemic stress responses ${ }^{[41]}$. Evaluation of the mean changes of hippocampal volumes shows that the hippocampus is about $4 \%-5 \%$ smaller in patients with MDD than in healthy controls ${ }^{[42]}$. Using in situ DNA end-labeling demonstrated slightly increased rate of apoptosis in the dentate gyrus, CA1 and CA3 areas of the hippocampus in depressed patients ${ }^{[43]}$. In another postmortem study, Stockmeier et al found that hippocampal sections from depressed patients shrank more than sections from control subjects after histologic processing ${ }^{[44]}$. Hence, it suggests that both apoptosis and atrophy may occur in depression. BDNF clearly has a role in survival of neurons during hippocampal development, and this may relate to its putative role in depression. Decreased levels of BDNF may contribute to the atrophy of hippocampus that has been observed in depressed patients. Moreover, consistent data suggest that serum BDNF levels are reduced in depressed patients and are negatively correlated with depression severity while antidepressant treatment restores the basal level of serum BDNF ${ }^{[45,46]}$. Decreased BDNF mRNA and protein levels, TrkB and cyclic adenosine monophosphate (cAMP) response element binding protein (CREB) have been found in postmortem brains of suicide victims ${ }^{[47]}$. Consistently, suicidal behavior has been associated with lower plasma BDNF level ${ }^{[48]}$. In postmortem hippocampal sections, BDNF expression has been observed to be increased in dentate gyrus and subgranular regions in subjects treated with antidepressants prior to death as compared with non-treated subjects ${ }^{[49]}$.

Postmortem studies have a number of limitations in assessing depression-related molecular or cell turnover. Particularly, it is difficult to rule out the effects of antidepressant medication and other drugs or factors that are not known to investigators. Therefore, animal models were applied to investigate clear depression-induced hippocampal changes. Stress is widely used as a model for mood disorders in experi- mental animals. A summary of studies demonstrate that many different types of acute (single stress) and chronic (7 to $21 \mathrm{~d}$ ) stress paradigms decrease the expression of BDNF in the hippocampus. In chronically stressed tree shrews, an animal model with high validity for depression, demonstrated reduced hippocampal volume and cell proliferation, and these alterations were prevented by tianeptine treatment ${ }^{[50]}$. Immobilization stress significant decreases BDNFmRNA expression in the major subfields of the hippocampus, with the greatest effects observed in the dentate gyrus granule cell layer. Subsequent work found that other types of stress, including unpredictable, footshock, swim stress, and maternal deprivation, also decreased the expression of BDNF in the hippocampus ${ }^{[51,52]}$. Genetic studies support the role of BDNF Val66Met polymorphism in depression ${ }^{[13,14]}$. In our previous study, BDNF ${ }^{\text {Met/Met }}$ mice have smaller hippocampal volumes and increased anxiety-related behaviors ${ }^{[19]}$. Moreover, music treatment could decrease anxiety state in $\mathrm{BDNF}^{\mathrm{Met} / \mathrm{Met}}$ mice by increasing BDNF levels in the hippocampus and prefrontal cortex ${ }^{[53]}$.

In contrast to the actions of stress, different classes of antidepressants significantly increased the expression of BDNF in the major subfields of the hippocampus, including the granule cell layer and the CA1 and CA3 pyramidal cell layers $^{[54]}$. Chronic pretreatment with antidepressants blocks the stress-induced decrease in BDNF mRNA expression in the hippocampus ${ }^{[55]}$. Distinct classes of antidepressants appear to regulate the BDNF gene through differential recruitment of individual BDNF promoters ${ }^{[56]}$. In addition, they appear to be capable of reversing the epigenetic shut down of the BDNF III and IV promoters caused by stress. Indeed, overexpression of histone deacetylases, which would derepress the epigenetic control of the BDNF promoters, is shown to upregulate hippocampal BDNF expression and exert antidepressant-like effects in animal models ${ }^{[57]}$. Furthermore, truncated TrkB overexpressing mice, which show reduced BDNF signaling, are resistant to the effects of antidepressants in the forced swim model of depression ${ }^{[58]}$. Several lines of evidence suggest that increased BDNF signaling is associated with antidepressantlike behaviors in rodent depression models. Specifically, Siuciak and colleagues were the first to report that short-term (6-7 d), microinfusion of BDNF into the dentate gyrus of hippocampus produces antidepressant-like effect in forced swim test (FST) and learned helpless (LH) ${ }^{[59]}$. Furthermore, a single intracerebroventricular injection of BDNF $(10 \mu \mathrm{g})$ produces similar changes in the modified rat FST ${ }^{[60]}$. Compared with the classical antidepressants, the effects of BDNF on these behaviors are long lasting ${ }^{[60,61]}$. Unfortunately, relatively high doses of BDNF need to be administered to achieve these behavioral responses. Taken together, these reports clearly implicate BDNF in both the pathogenesis of depression and as a target/ mediator of antidepressant action.

Several studies have suggested that normal BDNF signaling is both necessary and sufficient for antidepressant drug action. Antidepressant-induced tyrosine phosphorylation of TrkB does not induce activity of the extracellular signal-regulated kinase pathway, but does activate phospholipase- $\mathrm{C} \gamma$ signaling 
and lead to the phosphorylation of CREB, a major transcription factor directing gene expression of plasticity-related molecules ${ }^{[62]}$. In the hippocampus, increased activity of the BDNFCREB cascade results in antidepressive responses. Chronic, but not acute, administration of several different types of antidepressants, including SSRIs, a NE-selective reuptake inhibitor, a monoamine oxidase inhibitor, an atypical antidepressant, ECS, and lithium, increase the levels of CREB mRNA and immunoreactivity in hippocampus ${ }^{[5]}$. In situ hybridization and immunohistochemical analysis demonstrate that the expression of CREB is increased in the major cell layers of hippocampus (ie, CA3 and CA1 pyramidal and dentate gyrus granule cell layers). In contrast, chronic administration of nonantidepressant psychotropic drugs (ie, morphine, cocaine, and haloperidol) does not influence the expression of CREB in hippocampus, demonstrating the pharmacologic specificity of this effect for antidepressants. This does not imply that the cAMP system and CREB are not involved in the actions of these drugs. In fact, chronic opiate or psychostimulant treatments are reported to regulate CREB in striatum and locus coeruleus $^{[63]}$. This demonstrates that CREB is regulated in a region-specific manner depending on the neurotransmitter systems influenced by these psychotropic drug treatments.

Hippocampal overexpression of BDNF or CREB is capable of mimicking both the structural consequences of sustained antidepressant treatment as well as exerting antidepressantlike behavioral effects ${ }^{[61,64]}$. Indeed, activation of the cAMPCREB cascade results in increased neurogenesis of dentate granule cell progenitors, and increased dendritic length and branching ${ }^{[6]]}$. It is possible that CREB, a transcriptional activator of BDNF, recruits this neurotrophin to mediate its effects on structural plasticity. BDNF, in addition to being a target of CREB, can itself recruit this particular transcription factor by activating the mitogen-activated protein (MAP) kinase cascade $^{[66]}$, thus setting up a potential positive feed-back loop. Taken together, elevated BDNF-CREB, through their protective influences on vulnerable hippocampal neurons and their ability to directly promote structural reorganization, could result in repair of this region known to be damaged in depression. In addition, BDNF can alter neurotransmitter release and itself elicit an activation of postsynaptic neurons, and may thus have potential protective functional consequences on hippocampal circuitry known to be dysfunctional in depres$\operatorname{sion}^{[67]}$. A direct consequence of enhanced hippocampal function would be a restoration of the inhibitory control exerted on the stress response pathway of the HPA axis. In addition, the well-established role of BDNF and CREB in hippocampaldependent learning and memory may play a critical role in ameliorating the cognitive symptoms associated with depression $^{[67,68]}$. Therefore, reduced BDNF levels result in neuronal atrophy and cell death in the hippocampus whereas enhanced BDNF levels are associated with neurogenesis, cell survival and dendritic arborization. Thus, changes in hippocampal BDNF levels and the resulting downstream signaling pathways may play an essential role in regulating depression related behaviors.

\section{BDNF signaling in prefrontal cortex involved in depression}

Several lines of evidence suggest that the prefrontal cortex (PFC) is involved in the neuropathology of depression and the response to stress. Perhaps the most widely accepted division of PFC, based on anatomical connectivity and functional specialization, is the dorsolateral and ventromedial sectors ${ }^{[69]}$. The ventromedial prefrontal cortex (vmPFC) includes the ventral portion of the medial prefrontal cortex and medial portion of the orbital surface. Targets of vmPFC projections include the hypothalamus and periaqueductal gray, which mediate the visceral autonomic activity associated with emotion, and the ventral striatum, which signals reward and motivational value. In addition, vmPFC has dense reciprocal connections with the amygdala, which is involved in threat detection and fear conditioning ${ }^{[29]}$. By contrast, the dorsolateral prefrontal cortex (DLPFC), which includes portions of the middle and superior frontal gyri on the lateral surface of the frontal lobes, receives input from specific sensory cortices, and has dense interconnections with premotor areas, the frontal eye fields, and lateral parietal cortex ${ }^{[70]}$. The distinct patterns of connectivity in these two regions of PFC suggest disparate functionality. Indeed, DLPFC has primarily been associated with "cognitive" or "executive" functions, whereas vmPFC is largely ascribed "emotional" or "affective" functions.

Volumetric changes in the PFC in depression are similar to those described in the human hippocampus. The earliest functional imaging studies of depression compared the resting state brain activity (eg blood flow or glucose metabolism) of depressed patients with that of non-depressed comparison subjects. Results from these studies associate depression with abnormally high levels of vmPFC activity ${ }^{[71]}$, but abnormally low levels of DLPFC activity in resting brain activity ${ }^{[72]}$. Recent, a type of functional imaging study compares taskrelated brain activations (blood flow) of depressed patients to that of non-depressed comparison subjects. Data from these studies demonstrate that depressed patients exhibit greater task-related activation in DLPFC during tests of working memory and cognitive control when performance is matched to non-depressed subjects ${ }^{[73]}$. In light of the resting state data indicating DLPFC hypoactivity in depression, these results suggest dysfunction (or at least inefficiency) in the DLPFC of depressed patients. In sum, the functional imaging studies converge to suggest that depression is associated with seemingly opposite activity profiles in VmPFC and DLPFC. The vmPFC is hyperactive at rest, whereas the DLPFC is hypoactive at rest. These imaging data hint that an imbalance in vmPFC/DLPFC activity may contribute to depression. However, functional imaging data alone cannot adjudicate whether the abnormal activity profiles observed in vmPFC and DLPFC are a cause or consequence of the disorder.

In humans, postmortem studies have shown that both BDNF and TrkB levels are significantly decreased in the prefrontal cortex and hippocampus of suicide patients compared with controls and antidepressant therapy restores brain BDNF levels to the normal range ${ }^{[74]}$. In addition, there were signifi- 
cant decreases in the phosphorylation of TrkA and TrkB in both PFC and hippocampus of suicide subjects, whereas the phosphorylation of TrkC was decreased only in hippocampus without any change in $\mathrm{PFC}^{[75]}$. Stress is used as a model to study alterations of molecules and brain structure because mood disorders are often precipitated or exacerbated by acute or chronic stressful life events ${ }^{[76]}$. Repeated stress causes dendritic shortening in medial prefrontal cortex, as well as in hippocampus ${ }^{[77]}$. As with in the hippocampus, stress decrease BDNF levels in the PFC. Prenatally stressed rats had significantly less BDNF protein than nonstressed rats in PFC by Western blotting analysis ${ }^{[7]}$. Furthermore, Roth showed that infant maltreatment results in methylation of BDNF DNA through the lifespan to adulthood that dovetails reduced $B D N F$ gene expression in the adult PFC in rat ${ }^{[79]}$.

Many different antidepressants produce a modest increase in BDNF mRNA levels in PFC after a few days of treatment. BDNF mRNA and protein levels, as well as TrkB mRNA levels, were increased significantly in post-natal day 13 rats in hippocampus and PFC after escitalopram treatment as compared to control, but desipramine failed to increase either BDNF or TrkB, which suggests that SSRIs are able to positively modulate BDNF and TrkB expression, whereas desipramine is not able to. The failure of desipramine to positively modulate BDNF and TrkB expression in postnatal day 13 rats is consistent with the lack of efficacy of desipramine in children and adolescents ${ }^{[80]}$. The BDNF gene consists of four $5^{\prime}$ noncoding exons (I-IV) each with a separate promotor and one 3 ' exon (exon- $\mathrm{V}$ ) encoding the mature BDNF protein. The exon-III and IV BDNF promoters are regulated as immediate early genes. Previous work showed that BDNF-LTP is associated with rapid upregulation of exon-III specific and exon-V (total) BDNF mRNA. In PFC exon-III and exon-V BDNF mRNA levels were significantly elevated about 3- and 2-fold, respectively, after chronic but not acute fluoxetine treatment. In hippocampus, chronic fluoxetine administration led to a 2.5-fold increase in exon-III expression, but no significant change in exon- $\mathrm{V}$ expression. These studies demonstrate that chronic administration of fluoxetine leads to brain region specific upregulation of BDNF in the adult brain, which suggests that chronically administered antidepressants could promote BDNF-induced gene expression and synaptic plasticity in multiple brain regions.

Animals work shows that stress decreases phosphorylated CREB and antidepressants treatment increases the expression of phosphorylated CREB specifically in the hippocampus and prefrontal cortex ${ }^{[81]}$. Experimental studies demonstrated that chronic forced swim stress decreased the expression of phosphorylated-extracellular signal-regulated kinase 2 (p-ERK2), ERK1 and ERK2 in the hippocampus and prefrontal cortex in rats; fluoxetine reversed the stress-induced disruption of the p-ERK2, which is indicated by the increased level of the p-ERK2 in the hippocampus and prefrontal cortex in stressfluoxetine group compared to stress group, but exhibited no effect on the stress-induced decrease of the ERK1 and ERK2. It thus appears that the major effect of fluoxetine is to acti- vate the ERK and CREB to increase the levels of p-ERK2 and p-CREB, but not to increase the ERK and CREB expression ${ }^{[82]}$.

\section{BDNF signaling in nucleus accumbens involved in depression}

The NAc is a target of the mesolimbic dopamine system, which receives dopamine input from dopaminergic neurons in the ventral tegmental area (VTA) of the midbrain. Furthermore, the ventral striatum also has been noted to have extensive connections with the amygdala and the orbital, subgenual, and ventrolateral $\mathrm{PFC}^{[83]}$. The NAc, and its dopaminergic inputs, play critical roles in reward. Virtually all drugs of abuse increase dopaminergic transmission in the NAc, which partly mediates their rewarding effects ${ }^{[84]}$. On the other hand, the VTA neurons also innervate several other limbic structures, including the amygdala and limbic regions of neocortex. The relationship of VTA-NAc pathway to mood disorders requires further study, but it seems plausible that disturbances in this pathway would be related to abnormalities in hedonic tone and motivation ${ }^{[2]}$, which are central features of mania and depression. This is supported by the finding of decreased striatal response to happy stimuli associated with level of anhedonia in depressed subjects as well as the observation of increased striatal activity in mania ${ }^{[85]}$.

However, increasing BDNF levels in the NAc or VTA pathway produces depression-like phenotype and animals with a selective knockout of BDNF in the VTA are protected from the depressive effects produced by the social defeat stress ${ }^{[23]}$. At the same time, intra-VTA infusion of BDNF exerts a depression-like effect in the forced swim test, while blockade of BDNF action in the NAc, by use of viral-mediated overexpression of a dominant negative mutant of TrkB, causes an antidepressant-like effect in the same test ${ }^{[86]}$. These data suggest that BDNF plays opposite roles in the VTA-NAc circuit compared with in the hippocampal-prefrontal circuit.

While dysfunction of the VTA-NAc circuit is thought to be associated with depression, antidepressants have been postulated to reverse this dysfunction. In stark contrast to the effects of CREB-BDNF in the hippocampus, activation of the CREB-BDNF cascade in the VTA-NAc pathway results in pro-depressive like behavior ${ }^{[64]}$. Increased CREB activity in the NAc results in a phenotype characteristic of depression, including reduced reward experience or "anhedonia" and increased immobility in the forced swim test, symbolizing behavioral "despair" [87]. The effects of BDNF are thought to be mediated via up-regulating CREB expression, through activation of the MAPK/ERK pathway ${ }^{[66]}$. Thus BDNF, upstream of CREB, would recruit specific target genes with promoter CRE elements, and then affect the functioning of the VTANAc circuitry, resulting in a depression-like phenotype. CREB is known to positively modulate levels of dynorphin within the $N A c^{[88]}$, and this upregulation of the endogenous opioid dynorphin could mediate the pro-depressive effects of CREB. Although at present it is unknown if CREB induction in the NAc results in enhanced dynorphin release within the VTA, it has been hypothesized that such a change in the context of 
the VTA-NAc pathway could result in dysphoria and lack of pleasure seeking ${ }^{[89]}$. This raises the possibility that enhanced BDNF-CREB in the NAc may, through a regulation of opioid signalling, result in an anhedonic state thus contributing to the pro-depressive effects, whereas an abrogation of BDNF-CREB signalling in this region could have beneficial consequences on behavior and exert an antidepressant-like effect. The opposite behavioral response for infusion BDNF to hippocampus versus NAc suggests that depression and antidepressant effects are related to the functional consequences of different downstream regulation in different neuronal networks.

\section{BDNF signaling in amygdala involved in depression}

Recent years, the amygdala has emerged as the key forebrain structure mediating inborn and acquired emotional responses, as well as processing, interpreting, and integrating various aspects of biologically and/or emotionally important information $^{[90]}$. The amygdala is a complex structure that is comprised of many subnuclei. Two such units that have been particularly implicated in the control of emotional processes are the central nucleus $(\mathrm{CeA})$ and the basolateral amygdala (BLA). A prevailing view is that the BLA is responsible for emotional learning; receiving sensory information and acts as a site of conditioned and unconditioned stimulus associations and uses this learned information to control the activity of the CeA. In turn, the CeA acts as a 'controller of the brainstem', by using its widespread projections to the hypothalamus, midbrain reticular formation and brainstem to orchestrate behavioral, autonomic, and neuroendocrine responses. Different regions within the amygdala are specialized, on the basis of connections with cortical, hippocampal, thalamic, hypothalamic, and other subcortical structures, to detect objects salient to the prevailing emotional state, and to modulate an appropriate response ${ }^{[91,92]}$. Dysfunction in amygdala plasticity can be related theoretically to depression and bipolar disorder (BD) ${ }^{[91]}$. Neuroimaging studies of the amygdala in patients with BD are characterized by an interesting age-related dichotomy of findings. In adults, the predominant pattern is one of increased amygdala volume while in children and adolescents the reverse applies, which suggest that amygdala size may vary in relation to illness duration ${ }^{[93,94]}$. Resting state functional analyses have been largely limited to the adult population and are indicative of increased baseline amygdala activity which correlates positively with severity of depression ${ }^{[95]}$.

The link between stress and depression has long been observed, particularly at the clinical level, where chronic exposure to stressful life events has been associated with the development of depressive symptoms. Stress also causes structure changes in amygdala. Both acute and chronic stress increases spine synapse formation in amygdala ${ }^{[96]}$ but chronic stress decreases it in hippocampus ${ }^{[97]}$. Moreover, chronic stress for 21 days or longer impairs hippocampal-dependent cognitive function and enhances amygdala-dependent unlearned fear and fear conditioning ${ }^{[98]}$, which are consistent with the opposite effects of stress on hippocampal and amygdala struc- ture and suggest that the effect of chronic stress on dendritic remodeling is regionally specific.

BDNF mRNA increased in the amygdala $1 \mathrm{~h}$ after the final exposure to intermittent water immersion stress. Consistent with this, repeated intermittent social stress increased BDNF mRNA in the BLA and CeA but not in the medial amygdala (MeA) $2 \mathrm{~h}$ after termination of social stress. However, repeated restraint or acute social stress exposure reduced amygdala BDNF mRNA $24 \mathrm{~h}_{\text {later }}{ }^{[99,100]}$. This suggests that stress-induced changes in BDNF are transient in the amygdala. BDNF-overexpressing transgenic mice showed increased BLA spine density and increase anxiety-like behavior in open field test and elevated-plus maze test ${ }^{[101]}$. Furthermore, previous studies have reported that chronic stress-induced anxiety is accompanied by increases in dendritic area and spine density in the BLA ${ }^{[102,103]}$. This increase in the BLA spine density may be a reason for the increased amygdala size and functional output.

The amygdala has been the focus of a great deal of work in the anxiety, post-traumatic stress disorder (PTSD), and drug addiction fields, but has received relatively little attention in depression. It would be interesting to use behavioral tests that focus on the amygdala, as well as direct manipulations of specific proteins in the amygdala (eg, CREB and BDNF, among many others), to explore the role played by these circuits in depression and antidepressant action.

\section{Conclusions and future directions}

In summary, the data herein reviews the role of BDNF in critical neural networks underlying depression/stress and antidepressant treatment (Table 1). The main studies suggest four points: First, depression or stress could reduce BDNF levels in the hippocampus and PFC; Second, depression or stress induces dentritic atrophy in the hippocampus and PFC, while leads to increased spine density in the BLA; Third, successful antidepressant treatments increase BDNF levels in the hippocampus and PFC and inhibit depression-like behavior; Fourth, BDNF levels increase in the BLA and NAc produce anxiety-like or depression-like phenotype. Thus the diverse roles of BDNF in depression depend on its location in the neural circuitry, namely, in the hippocampus and PFC BDNF inhibits depressive symptoms and whereas in the NAc and amygdala facilitates depression-like or anxiety-like symptoms.

Regardless of the role of BDNF in the etiology of depression

Table 1. Regulation of BDNF by depression/stress, and antidepressant treatment in different brain regions.

\begin{tabular}{lcc}
\hline Brain regions & Depression/Stress & Antidepressant \\
\hline Hippocampus & BDNF $\downarrow$ & BDNF $\uparrow$ \\
PFC & BDNF $\downarrow$ & $B D N F \uparrow$ \\
NAc & BDNF $\uparrow$ & BDNF $\downarrow$ \\
Amygdala (BLA) & BDNF $\uparrow$ & - \\
\hline
\end{tabular}

Symbols: $\downarrow$, decrease; $\uparrow$, increase; _, inconsistent. 
and in the mechanism of action of current antidepressants, advances in understanding how specific molecular mechanisms within the hippocampus, PFC, NAc, amygdala, and other brain regions regulate mood will provide novel avenues. A major need of future research is to better define the detailed circuitry of the numerous and diverse molecular pathways in these brain regions.

\section{Acknowledgements}

This study was supported by National Natural Science Foundation of China (No 30725020 and 30700258), China Postdoctoral Science Foundation (No 20090461232 and 200901006) and Independent Innovation Foundation of Shandong University (№ 2009TS126 and 2010TS088).

\section{References}

1 Briley M, Moret C. Present and future anxiolytics. IDrugs 2000; 3: 695-9.

2 Nestler EJ, Barrot M, DiLeone RJ, Eisch AJ, Gold SJ, Monteggia LM. Neurobiology of depression. Neuron 2002; 34: 13-25.

3 Fava M, Kendler KS. Major depressive disorder. Neuron 2000; 28: 335-41.

4 Burmeister M. Basic concepts in the study of diseases with complex genetics. Biol Psychiatry 1999; 45: 522-32.

5 Belmaker RH, Agam G. Major depressive disorder. N Engl J Med 2008; 358: 55-68.

6 Morilak DA, Frazer A. Antidepressants and brain monoaminergic systems: a dimensional approach to understanding their behavioural effects in depression and anxiety disorders. Int J Neuropsychopharmacol 2004; 7: 193-218.

7 Duman RS, Malberg J, Nakagawa S, D'Sa C. Neuronal plasticity and survival in mood disorders. Biol Psychiatry 2000; 48: 732-9.

8 Altar CA. Neurotrophins and depression. Trends Pharmacol Sci 1999; 20: 59-61.

9 Cunha AB, Frey BN, Andreazza AC, Goi JD, Rosa AR, Goncalves CA, et al. Serum brain-derived neurotrophic factor is decreased in bipolar disorder during depressive and manic episodes. Neurosci Lett 2006; 398: 215-9.

10 Palomino A, Vallejo-IIlarramendi A, Gonzalez-Pinto A, Aldama A, Gonzalez-Gomez C, Mosquera F, et al. Decreased levels of plasma BDNF in first-episode schizophrenia and bipolar disorder patients. Schizophr Res 2006; 86: 321-2.

11 Chen ZY, Patel PD, Sant G, Meng CX, Teng KK, Hempstead BL, et al. Variant brain-derived neurotrophic factor (BDNF) (Met66) alters the intracellular trafficking and activity-dependent secretion of wild-type BDNF in neurosecretory cells and cortical neurons. J Neurosci 2004; 24: 4401-11.

12 Egan MF, Kojima M, Callicott JH, Goldberg TE, Kolachana BS, Bertolino A, et al. The BDNF val66met polymorphism affects activitydependent secretion of BDNF and human memory and hippocampal function. Cell 2003; 112: 257-69.

13 Post RM. Role of BDNF in bipolar and unipolar disorder: clinical and theoretical implications. J Psychiatr Res 2007; 41: 979-90.

14 Monteggia LM, Luikart B, Barrot M, Theobold D, Malkovska I, Nef S, et al. Brain-derived neurotrophic factor conditional knockouts show gender differences in depression-related behaviors. Biol Psychiatry 2007; 61: 187-97.

15 Lang UE, Hellweg R, Kalus P, Bajbouj M, Lenzen KP, Sander T, et al. Association of a functional BDNF polymorphism and anxiety-related personality traits. Psychopharmacology (Berl) 2005; 180: 95-9.

16 Kunugi $\mathrm{H}$, Ueki $\mathrm{A}$, Otsuka $\mathrm{M}$, Isse $\mathrm{K}$, Hirasawa $\mathrm{H}$, Kato $\mathrm{N}$, et al. A novel polymorphism of the brain-derived neurotrophic factor (BDNF) gene associated with late-onset Alzheimer's disease. Mol Psychiatry 2001; 6: 83-6.

17 Mirowska-Guzel D, Mach A, Gromadzka G, Czlonkowski A, Czlonkowska A. BDNF A196G and C270T gene polymorphisms and susceptibility to multiple sclerosis in the Polish population. Gender differences. J Neuroimmunol 2008; 193: 170-2.

18 Duman RS, Monteggia LM. A neurotrophic model for stress-related mood disorders. Biol Psychiatry 2006; 59: 1116-27.

19 Chen ZY, Jing D, Bath KG, leraci A, Khan T, Siao CJ, et al. Genetic variant BDNF (Val66Met) polymorphism alters anxiety-related behavior. Science 2006; 314: 140-3.

20 Yu H, Wang Y, Pattwell S, Jing D, Liu T, Zhang Y, et al. Variant BDNF Val66Met polymorphism affects extinction of conditioned aversive memory. J Neurosci 2009; 29: 4056-64.

21 Kozisek ME, Middlemas D, Bylund DB. Brain-derived neurotrophic factor and its receptor tropomyosin-related kinase $B$ in the mechanism of action of antidepressant therapies. Pharmacol Ther 2008; 117: 30-51.

22 Pittenger C, Duman RS. Stress, depression, and neuroplasticity: a convergence of mechanisms. Neuropsychopharmacology 2008; 33: 88-109.

23 Berton O, Nestler EJ. New approaches to antidepressant drug discovery: beyond monoamines. Nat Rev Neurosci 2006; 7: 137-51.

24 Groves JO. Is it time to reassess the BDNF hypothesis of depression? Mol Psychiatry 2007; 12: 1079-88.

25 Martinowich K, Manji H, Lu B. New insights into BDNF function in depression and anxiety. Nat Neurosci 2007; 10: 1089-93.

26 Castren E, Voikar V, Rantamaki T. Role of neurotrophic factors in depression. Curr Opin Pharmacol 2007; 7: 18-21.

27 Russo-Neustadt AA, Chen MJ. Brain-derived neurotrophic factor and antidepressant activity. Curr Pharm Des 2005; 11: 1495-510.

28 Berton O, McClung CA, Dileone RJ, Krishnan V, Renthal W, Russo SJ, et al. Essential role of BDNF in the mesolimbic dopamine pathway in social defeat stress. Science 2006; 311: 864-8.

29 Ongur D, Price JL. The organization of networks within the orbital and medial prefrontal cortex of rats, monkeys and humans. Cereb Cortex 2000; 10: 206-19.

30 Chamberlain SR, Sahakian BJ. The neuropsychology of mood disorders. Curr Psychiatry Rep 2006; 8: 458-63.

31 Davidson RJ, Abercrombie H, Nitschke JB, Putnam K. Regional brain function, emotion and disorders of emotion. Curr Opin Neurobiol 1999; 9: 228-34.

32 Ressler KJ, Nemeroff CB. Role of serotonergic and noradrenergic systems in the pathophysiology of depression and anxiety disorders. Depress Anxiety 2000; 12: 2-19.

33 Sheline YI, Wang PW, Gado MH, Csernansky JG, Vannier MW. Hippocampal atrophy in recurrent major depression. Proc Natl Acad Sci USA 1996; 93: 3908-13.

34 Sheline YI, Gado MH, Kraemer HC. Untreated depression and hippocampal volume loss. Am J Psychiatry 2003; 160: 1516-8.

35 Ongur D, Drevets WC, Price JL. Glial reduction in the subgenual prefrontal cortex in mood disorders. Proc Natl Acad Sci USA 1998; 95: 13290-5.

36 Baumann B, Danos P, Krell D, Diekmann S, Leschinger A, Stauch R, et al. Reduced volume of limbic system-affiliated basal ganglia in mood disorders: preliminary data from a postmortem study. J Neuropsychiatry Clin Neurosci 1999; 11: 71-8.

37 Lacerda AL, Nicoletti MA, Brambilla P, Sassi RB, Mallinger AG, Frank 
E, et al. Anatomical MRI study of basal ganglia in major depressive disorder. Psychiatry Res 2003; 124: 129-40.

38 Frodl T, Meisenzahl EM, Zetzsche T, Born C, Jager M, Groll C, et al. Larger amygdala volumes in first depressive episode as compared to recurrent major depression and healthy control subjects. Biol Psychiatry 2003; 53: 338-44.

39 Siegle GJ, Konecky RO, Thase ME, Carter CS. Relationships between amygdala volume and activity during emotional information processing tasks in depressed and never-depressed individuals: an fMRI investigation. Ann N Y Acad Sci 2003; 985: 481-4.

40 LaBar KS, Cabeza R. Cognitive neuroscience of emotional memory. Nat Rev Neurosci 2006; 7: 54-64.

41 Jacobson L, Sapolsky R. The role of the hippocampus in feedback regulation of the hypothalamic-pituitary-adrenocortical axis. Endocr Rev 1991; 12: 118-34.

42 Videbech P, Ravnkilde B. Hippocampal volume and depression: a meta-analysis of MRI studies. Am J Psychiatry 2004; 161: 1957-66.

43 Lucassen PJ, Muller MB, Holsboer F, Bauer J, Holtrop A, Wouda J, et al. Hippocampal apoptosis in major depression is a minor event and absent from subareas at risk for glucocorticoid overexposure. Am J Pathol 2001; 158: 453-68.

44 Stockmeier CA, Mahajan GJ, Konick LC, Overholser JC, Jurjus GJ, Meltzer HY, et al. Cellular changes in the postmortem hippocampus in major depression. Biol Psychiatry 2004; 56: 640-50.

45 Aydemir C, Yalcin ES, Aksaray S, Kisa C, Yildirim SG, Uzbay T, et al. Brain-derived neurotrophic factor (BDNF) changes in the serum of depressed women. Prog Neuropsychopharmacol Biol Psychiatry 2006; 30: 1256-60.

46 Lee BH, Kim H, Park SH, Kim YK. Decreased plasma BDNF level in depressive patients. J Affect Disord 2007; 101: 239-44.

47 Dwivedi Y, Rao JS, Rizavi HS, Kotowski J, Conley RR, Roberts RC, et al. Abnormal expression and functional characteristics of cyclic adenosine monophosphate response element binding protein in postmortem brain of suicide subjects. Arch Gen Psychiatry 2003; 60: 273-82.

48 Kim YK, Lee HP, Won SD, Park EY, Lee HY, Lee BH, et al. Low plasma BDNF is associated with suicidal behavior in major depression. Prog Neuropsychopharmacol Biol Psychiatry 2007; 31: 78-85.

49 Chen B, Dowlatshahi D, MacQueen GM, Wang JF, Young LT. Increased hippocampal BDNF immunoreactivity in subjects treated with antidepressant medication. Biol Psychiatry 2001; 50: 260-5.

50 Czeh B, Michaelis T, Watanabe T, Frahm J, de Biurrun G, van Kampen M, et al. Stress-induced changes in cerebral metabolites, hippocampal volume, and cell proliferation are prevented by antidepressant treatment with tianeptine. Proc Natl Acad Sci USA 2001; 98: 12796-801.

51 Rasmusson AM, Shi L, Duman R. Downregulation of BDNF mRNA in the hippocampal dentate gyrus after re-exposure to cues previously associated with footshock. Neuropsychopharmacology 2002; 27: 133-42.

52 Roceri M, Cirulli F, Pessina C, Peretto P, Racagni G, Riva MA. Postnatal repeated maternal deprivation produces age-dependent changes of brain-derived neurotrophic factor expression in selected rat brain regions. Biol Psychiatry 2004; 55: 708-14.

53 Li WJ, Yu H, Yang JM, Gao J, Jiang H, Feng M, et al. Anxiolytic effect of music exposure on BDNFMet/Met transgenic mice. Brain Res 2010; 1347: 71-9.

54 Russo-Neustadt AA, Alejandre H, Garcia C, Ivy AS, Chen MJ. Hippocampal brain-derived neurotrophic factor expression following treatment with reboxetine, citalopram, and physical exercise. Neuropsychopharmacology 2004; 29: 2189-99.
55 Nibuya M, Morinobu S, Duman RS. Regulation of BDNF and trkB mRNA in rat brain by chronic electroconvulsive seizure and antidepressant drug treatments. J Neurosci 1995; 15: 7539-47.

56 Dias BG, Banerjee SB, Duman RS, Vaidya VA. Differential regulation of brain derived neurotrophic factor transcripts by antidepressant treatments in the adult rat brain. Neuropharmacology 2003; 45: 553-63.

57 Tsankova NM, Berton O, Renthal W, Kumar A, Neve RL, Nestler EJ. Sustained hippocampal chromatin regulation in a mouse model of depression and antidepressant action. Nat Neurosci 2006; 9: 51925.

58 Saarelainen T, Hendolin P, Lucas G, Koponen E, Sairanen M, MacDonald $\mathrm{E}$, et al. Activation of the TrkB neurotrophin receptor is induced by antidepressant drugs and is required for antidepressantinduced behavioral effects. J Neurosci 2003; 23: 349-57.

59 Siuciak JA, Lewis DR, Wiegand SJ, Lindsay RM. Antidepressantlike effect of brain-derived neurotrophic factor (BDNF). Pharmacol Biochem Behav 1997; 56: 131-7.

60 Hoshaw BA, Malberg JE, Lucki I. Central administration of IGF-I and BDNF leads to long-lasting antidepressant-like effects. Brain Res 2005; 1037: 204-8.

61 Shirayama Y, Chen AC, Nakagawa S, Russell DS, Duman RS. Brainderived neurotrophic factor produces antidepressant effects in behavioral models of depression. J Neurosci 2002; 22: 3251-61.

62 Duman RS. Pathophysiology of depression: the concept of synaptic plasticity. Eur Psychiatry 2002; 17: 306-10.

63 Widnell KL, Russell DS, Nestler EJ. Regulation of expression of cAMP response element-binding protein in the locus coeruleus in vivo and in a locus coeruleus-like cell line in vitro. Proc Natl Acad Sci USA 1994; 91: 10947-51.

64 Malberg JE, Blendy JA. Antidepressant action: to the nucleus and beyond. Trends Pharmacol Sci 2005; 26: 631-8.

65 Fujioka T, Fujioka A, Duman RS. Activation of cAMP signaling facilitates the morphological maturation of newborn neurons in adult hippocampus. J Neurosci 2004; 24: 319-28.

66 Arthur JS, Fong AL, Dwyer JM, Davare M, Reese E, Obrietan K, et al. Mitogen- and stress-activated protein kinase 1 mediates cAMP response element-binding protein phosphorylation and activation by neurotrophins. J Neurosci 2004; 24: 4324-32.

67 Tyler WJ, Perrett SP, Pozzo-Miller LD. The role of neurotrophins in neurotransmitter release. Neuroscientist 2002; 8: 524-31.

68 Mizuno K, Giese KP. Hippocampus-dependent memory formation: do memory type-specific mechanisms exist? J Pharmacol Sci 2005; 98: 191-7.

69 Zald DH. Orbital versus dorsolateral prefrontal cortex: anatomical insights into content versus process differentiation models of the prefrontal cortex. Ann N Y Acad Sci 2007; 1121: 395-406.

70 Barbas $\mathrm{H}$. Connections underlying the synthesis of cognition, memory, and emotion in primate prefrontal cortices. Brain Res Bull 2000; 52: 319-30.

71 Greicius MD, Flores BH, Menon V, Glover GH, Solvason HB, Kenna $\mathrm{H}$, et al. Resting-state functional connectivity in major depression: abnormally increased contributions from subgenual cingulate cortex and thalamus. Biol Psychiatry 2007; 62: 429-37.

72 Galynker II, Cai J, Ongseng F, Finestone H, Dutta E, Serseni D. Hypofrontality and negative symptoms in major depressive disorder. J Nucl Med 1998; 39: 608-12.

73 Wagner G, Sinsel E, Sobanski T, Kohler S, Marinou V, Mentzel HJ, et al. Cortical inefficiency in patients with unipolar depression: an event-related FMRI study with the Stroop task. Biol Psychiatry 2006; 59: 958-65. 
74 Castren E. Neurotrophic effects of antidepressant drugs. Curr Opin Pharmacol 2004; 4: 58-64.

75 Dwivedi Y, Rizavi HS, Zhang H, Mondal AC, Roberts RC, Conley RR, et al. Neurotrophin receptor activation and expression in human postmortem brain: effect of suicide. Biol Psychiatry 2009; 65: 31928.

76 Brown J, Cooper-Kuhn CM, Kempermann G, Van Praag H, Winkler J, Gage $\mathrm{FH}$, et al. Enriched environment and physical activity stimulate hippocampal but not olfactory bulb neurogenesis. Eur J Neurosci 2003; 17: 2042-6.

77 Brown SM, Henning S, Wellman CL. Mild, short-term stress alters dendritic morphology in rat medial prefrontal cortex. Cereb Cortex 2005; 15: 1714-22.

78 Burton CL, Chatterjee D, Chatterjee-Chakraborty M, Lovic V, Grella $\mathrm{SL}$, Steiner $\mathrm{M}$, et al. Prenatal restraint stress and motherless rearing disrupts expression of plasticity markers and stress-induced corticosterone release in adult female Sprague-Dawley rats. Brain Res 2007; 1158: 28-38.

79 Roth TL, Lubin FD, Funk AJ, Sweatt JD. Lasting epigenetic influence of early-life adversity on the BDNF gene. Biol Psychiatry 2009; 65: 760-9.

80 Kozisek ME, Middlemas D, Bylund DB. The differential regulation of BDNF and TrkB levels in juvenile rats after four days of escitalopram and desipramine treatment. Neuropharmacology 2008; 54: 251-7.

81 Sairanen M, O'Leary OF, Knuuttila JE, Castren E. Chronic antidepressant treatment selectively increases expression of plasticityrelated proteins in the hippocampus and medial prefrontal cortex of the rat. Neuroscience 2007; 144: 368-74.

82 Qi X, Lin W, Li J, Li H, Wang W, Wang D, et al. Fluoxetine increases the activity of the ERK-CREB signal system and alleviates the depressive-like behavior in rats exposed to chronic forced swim stress. Neurobiol Dis 2008; 31: 278-85.

83 Price JL, Carmichael ST, Drevets WC. Networks related to the orbital and medial prefrontal cortex; a substrate for emotional behavior? Prog Brain Res 1996; 107: 523-36.

84 Koob GF, Sanna PP, Bloom FE. Neuroscience of addiction. Neuron 1998; 21: 467-76.

85 Keedwell PA, Andrew C, Williams SC, Brammer MJ, Phillips ML. The neural correlates of anhedonia in major depressive disorder. Biol Psychiatry 2005; 58: 843-53.

86 Eisch AJ, Bolanos CA, de Wit J, Simonak RD, Pudiak CM, Barrot M, et al. Brain-derived neurotrophic factor in the ventral midbrain-nucleus accumbens pathway: a role in depression. Biol Psychiatry 2003; 54: 994-1005.

87 Pliakas AM, Carlson RR, Neve RL, Konradi C, Nestler EJ, Carlezon WA, Jr. Altered responsiveness to cocaine and increased immobility in the forced swim test associated with elevated cAMP response elementbinding protein expression in nucleus accumbens. J Neurosci 2001; 21: 7397-403.

88 Carlezon WA Jr, Thome J, Olson VG, Lane-Ladd SB, Brodkin ES, Hiroi
N, et al. Regulation of cocaine reward by CREB. Science 1998; 282 : 2272-5.

89 Nestler EJ, Carlezon WA Jr. The mesolimbic dopamine reward circuit in depression. Biol Psychiatry 2006; 59: 1151-9.

90 Phelps EA, LeDoux JE. Contributions of the amygdala to emotion processing: from animal models to human behavior. Neuron 2005; 48: 175-87.

91 Quiroz JA, Singh J, Gould TD, Denicoff KD, Zarate CA, Manji HK. Emerging experimental therapeutics for bipolar disorder: clues from the molecular pathophysiology. Mol Psychiatry 2004; 9: 756-76.

92 Yaniv D, Desmedt A, Jaffard R, Richter-Levin G. The amygdala and appraisal processes: stimulus and response complexity as an organizing factor. Brain Res Brain Res Rev 2004; 44: 179-86.

93 Brambilla P, Harenski K, Nicoletti M, Sassi RB, Mallinger AG, Frank $\mathrm{E}$, et al. MRI investigation of temporal lobe structures in bipolar patients. J Psychiatr Res 2003; 37: 287-95.

94 Frangou S. The maudsley bipolar disorder project. Epilepsia 2005; 46: 19-25.

95 Mah L, Zarate CA Jr, Singh J, Duan YF, Luckenbaugh DA, Manji HK, et al. Regional cerebral glucose metabolic abnormalities in bipolar II depression. Biol Psychiatry 2007; 61: 765-75.

96 Vyas A, Mitra R, Shankaranarayana Rao BS, Chattarji S. Chronic stress induces contrasting patterns of dendritic remodeling in hippocampal and amygdaloid neurons. J Neurosci 2002; 22: 6810-8.

97 Pawlak R, Rao BS, Melchor JP, Chattarji S, McEwen B, Strickland $\mathrm{S}$. Tissue plasminogen activator and plasminogen mediate stressinduced decline of neuronal and cognitive functions in the mouse hippocampus. Proc Natl Acad Sci USA 2005; 102: 18201-6.

98 Conrad CD, LeDoux JE, Magarinos AM, McEwen BS. Repeated restraint stress facilitates fear conditioning independently of causing hippocampal CA3 dendritic atrophy. Behav Neurosci 1999; 113: 902-13.

99 Pizarro JM, Lumley LA, Medina W, Robison CL, Chang WE, Alagappan $A$, et al. Acute social defeat reduces neurotrophin expression in brain cortical and subcortical areas in mice. Brain Res 2004; 1025: 10-20.

100 Fanous S, Hammer RP Jr, Nikulina EM. Short- and long-term effects of intermittent social defeat stress on brain-derived neurotrophic factor expression in mesocorticolimbic brain regions. Neuroscience 2010; 167: 598-607.

101 Govindarajan A, Rao BS, Nair D, Trinh M, Mawjee N, Tonegawa S, et al. Transgenic brain-derived neurotrophic factor expression causes both anxiogenic and antidepressant effects. Proc Natl Acad Sci USA 2006; 103: 13208-13.

102 Vyas A, Pillai AG, Chattarji S. Recovery after chronic stress fails to reverse amygdaloid neuronal hypertrophy and enhanced anxiety-like behavior. Neuroscience 2004; 128: 667-73.

103 Mitra R, Jadhav S, McEwen BS, Vyas A, Chattarji S. Stress duration modulates the spatiotemporal patterns of spine formation in the basolateral amygdala. Proc Natl Acad Sci USA 2005; 102: 9371-6. 